\title{
VISUALIZING MULTILAYERED GEOSPATIAL DATA IN VIRTUAL REALITY TO ASSESS PUBLIC LIGHTING
}

\author{
M. Spur ${ }^{1}$, N. Houel ${ }^{2}$, V. Tourre ${ }^{1}$, \\ ${ }^{1}$ AAU, Centrale Nantes, Nantes, France — (maxim.spur, vincent.tourre)@ec-nantes.fr \\ ${ }^{2}$ AAU, École Nationale Supérieure d'Architecture de Nantes, Nantes, France — nicolas.houel@ crenau.archi.fr
}

Commission IV, WG IV/9

KEY WORDS: Geovisualization, Urban Data, Multilayer, Virtual Reality, User Study, Public Lighting, Public Participation

\begin{abstract}
:
With the improvement and proliferation of virtual reality devices, their use for research and professional activity is broadening, fostering the advent of the field of immersive analytics, as is their acceptance among consumers. Other than the heightened sense of immersion into visualized data they provide, they also make displays of much larger apparent size and different positioning practical than what would be possible otherwise. Drawing on these benefits, we implemented a development of Multiple and Coordinated Displays (MCVs) for geovisualization that stacks different layers of data above each other, tilted for legibility. In a formal experiment, we evaluated it and two other, comparable MCV methods implemented in VR for their usefulness in analyzing public perception and soliciting public feedback regarding urban street lighting. In that field, the direction has recently been shifting from purely systemic development to a participatory approach, thus our investigation was into how a system like this could facilitate participation that can yield actionable results. Previous analysis of interaction data and usability questionnaires reveals preferences for certain systems depending on user characteristics, with the stack system showing a slight advantage over a grid of layers and especially over temporal multiplexing. We show that regardless of MCV variation, participants were able to analyze and provide feedback on public lighting situations that can directly contribute to urbanist work. The MCV approach further aided in understanding their choices, as eye-tracking allowed us to analyze attention to individual data layers.
\end{abstract}

\section{INTRODUCTION}

Virtual reality devices have in recent years proliferated even into the consumer market, and keep making large steps to becoming more usable and acceptable not just for video games, but also for an increasing number of professional and scientific applications (Angulo, 2015, Cavallo et al., 2019), particularly in geovisualization (Çöltekin et al., 2016, Šašinka et al., 2019).

Their large field of view and stereoscopic presentation, along with their inherently immersive nature helped the field of immersive analytics (Dwyer et al., 2018) to emerge, which is now finding applications in a growing number of domains ( $\mathrm{Lu}$ et al., 2016, Cordeil et al., 2019), often being able to show otherwise hard to visualize data (Hurter et al., 2018).

Building on these advantages a virtual workspace can provide, we continue investigating an immersive implementation (fig. 1) (Spur et al., 2020) of three different multiple and coordinated view (MCV) systems (Roberts, 2007) for visualizing multilayered geospatial data, going beyond the goal of just evaluating their usability, and trying to validating the applicability of such VR tools in soliciting useful citizen participation in answering questions from urbanism.

Two of these MCVs are larger versions of classical systems - a grid and a "blitting" display, which switches between layers interactively - made more immersive by being in VR, while the third - a tilted stack a of layers - would be impractical outside of a virtual environment, and aims to improve on the MCV metaphor by keeping layers rendered at a perceptively large size closer together, minimizing the distance between them.

In this work we also focus on our approach of integrating urban studies directly into geovisualization research. For this goal, we developed a use case in urban studies concerning the perception and participatory improvement of public lighting.

The practice of artificial lighting is based on technical and political choices developed over several centuries. Since the 1970s, it has been regularly questioned, first by observing the worsening of light nuisances which disrupt astronomical work, then by the increase in energy consumption due to the expansion of the public lighting fleet.

In an unprecedented situation, partial or total extinguishing of lighting public is now considered for towns. Some small French municipalities have opted for this practice which remains relatively complex to imagine in the dense urban fabric of a metropolis, particularly because of the intensity of nocturnal work shifts and personal practices. Where public lighting has been built in a systemic way, we now envisage its evolution through an empirical approach based on the acquisition of sensitive data from users of the public space at night.

By asking participants in a formal study to choose areas they think need improvement in terms of urban lighting, we attempted to also answer these questions: how does the public judge artificial lighting practices? What data do they consult to make these judgements? How to best present this data?

By way of splitting the presented data into separate layers and distributing them physically or temporally through MCVs, eye tracking technology allowed us to meter attention to individual data layers, and attempting to correlate this visual attention to the written answers participants provided about their choices. 


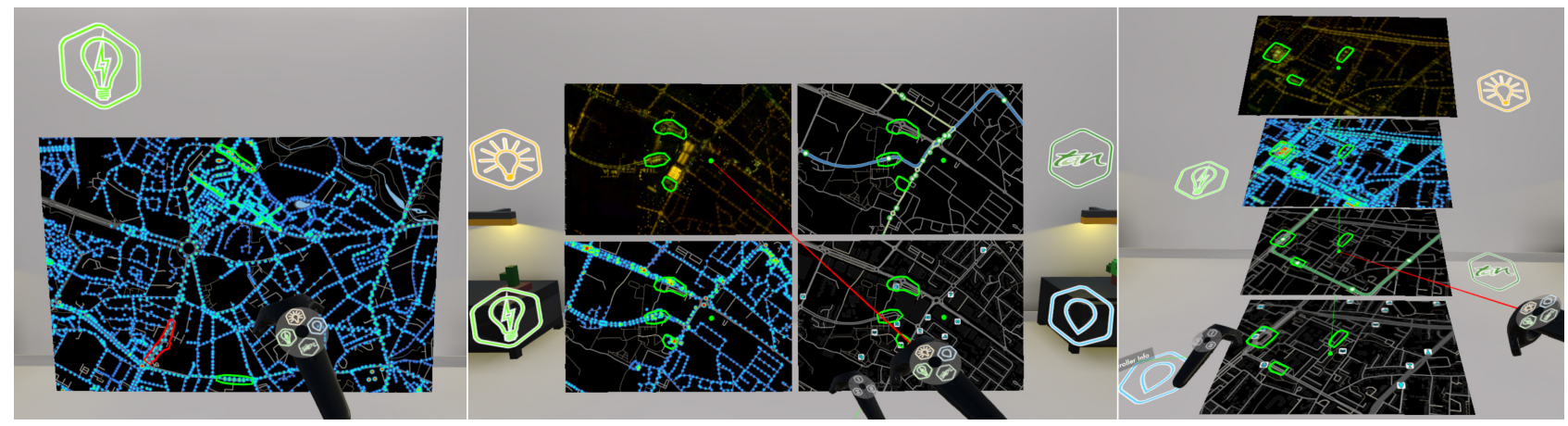

Figure 1. The blitting, grid, and stack MCV systems, and the controllers used to navigate map layers and the interface.

\section{RELATED WORK}

\subsection{Immersive Analytics}

Immersive analytics (Dwyer et al., 2018) is a recent field that emerged from enhancing visual analytics (Chandler et al., 2015) with newly available immersive AR and VR technologies, and aims to enhance the visualization of data from diverse domains not just through potentially superior means of displaying it stereoscopically and freely utilizing the vast virtual space available, but by literally and, hopefully figuratively, immersing a user into the presented data.

A recent survey through the field provides an insight into its many facets (Fonnet, Prié, 2019), and some advances have been made specifically in relation to exploring urban environments, however usually by visualizing 3D city models with some added information (Chen et al., 2017b, Berger, Bill, 2019), or utilizing the $3 \mathrm{D}$ environment to analyze one aspect of urban data from a new perspective (Filho et al., 2019).

\subsection{Urban Data Visualization}

3D city models have been utilized in non-immersive applications for urban data visualization, particularly when the threedimensional nature of the built environment plays a crucial role in what is to be evaluated, such as insolation (Freitas et al., 2015) and visibility (Ferreira et al., 2015, Ortner et al., 2016).

Using 3D models of cities can result in problematic occlusion of geometry, which can be addressed specifically, often through manipulating that geometry itself (Chen et al., 2017a, Trapp et al., 2019). Not all applications require full 3D visualization, though, in part because of problems like these, and much of cutting-edge research in urban data visualization remains purely in the domain of 2D maps (Zhen et al., 2020). While these implementations can still be conducive to visual analytics (Karduni et al., 2017), the immersion from the aforementioned technology is usually not utilized in these cases.

As the amount and complexity of data that needs to be visualized in urban environments increases, what can be displayed in one map may quickly reach the limits of what is feasible with cartographic design or even practical to display, considering the limitations of visual channels available to process the information (Munzner, 2014). Thus, the case can be made to instead split the relevant data into individual layers and display them separately, but coherently (Spur et al., 2017).

\subsection{Multiple and Coordinated Views}

Multiple and coordinated views (MCVs) (Roberts, 2007) are an especially appropriate method to deal with multilayered geospatial data. Other methods, such as lenses and overlays have been researched for two-layer comparison tasks in cartography (Lobo et al., 2015), but MCVs can easily handle larger numbers of layers, and remain useful. As their name implies, interactions are coordinated, or linked between them: zooming or panning in one layer while e.g. searching for a feature also moves all other layers, so attention can immediately be switched to another to quickly examine a region under different aspects.

With these separate views of individual layers taking up more visual space than a single one combining them, immersive systems offer a solution through their virtually limitless visual space, and the call for them to be thus utilized in immersive analytics (Knudsen, Carpendale, 2017) is beginning to get answered. AR systems can project virtual screens of information anywhere in a real room (Mahmood et al., 2018, Cavallo et al., 2019), and VR is able to construct any desired configuration.

\subsection{Immersive MCVs for Urban Data Visualization}

Combining these fields of immersive analytics of urban data using MCVs, a system has been implemented in VR that introduced vertical stacking of multiple data layers (Spur et al., 2020), and compared it against more common types of MCVs - a grid of layers, and a single view showing one layer at a time, but which can be switched by the user to show any other layer (fig. 1).

A formal study assessing the usability and preference of each of these MCV variations showed how user characteristics, such as their experience with 3D video games, and in particular their saccadic behavior as revealed through eye tracking are predictive of the speed and ease with which they are used, which is also directly linked to their stated preferences. The broad distribution of these characteristics among the participants showed that there was no "best" system overall, but instead that given a specific type of user, the appropriate variation had measurable advantages over the others.

In this work we go beyond the low-level analysis of these variations against each other, and look instead at how the general concept of immersive MCVs as implemented here can be useful for actual public participation studies involving multilayered geospatial data.

\subsection{Public lighting}

For a community, public lighting is a tool used to meet several objectives: securing goods and people, ensuring comfortable 
mobility, promoting heritage and landscapes, and accompanying nocturnal urban activities. In the current conception model of nocturnal ambiances used by teams of decision-makers, planners and installers, the users of a public lighting service, which evolves within nocturnal ambiances that were built for them, have no influence so far.

New considerations of energy factors have lead communities to greatly modify their approach to urban artificial lighting: it must now meet the traditional criteria regarding security, mobility and identity, while also committing to energy-saving and environment- and health-friendly strategies. This topic, which has recently been the main subject of one of the Great Debates initiated by municipal governments (Gambert, n.d.), seems to have become a general concern, with the consequences of increasing illumination having recently been observed and studied; their impact on fauna and flora (Kyba, Hölker, 2013) and on human health ((Zielinska-Dabkowska, 2018)) has been well documented.

The usually technical operation of public lighting thus finds itself facing more sensitive approaches. Its protocols in terms of political and technical decisions must now conform to questions of coexistence between species or of preservation of the night sky (Challéat, 2011) while also meeting recent regulatory requirements. The technical expertise thus encounters an increasing number of contradictions, e.g. illuminating a mobility space located at a water edge, staging the built and natural heritage regarding ecosystems darkness needs. Here, citizens could provide arbitration elements from their experiences, thus making them experts of the uses of territories at night. The problem remains the knowledge of the users about these contradictions. To potentially rank the political and technical choices associated with lighting, the users need to be informed clearly about various criteria to meet, for which an efficient way to provide information is needed, in order for them to contribute to improving public street lighting.

\section{IMPLEMENTATION}

The Unity 3D video game engine has found numerous use in visualization (Buyuksalih et al., 2017), and especially in geospatial and immersive analytics (Cordeil et al., 2019). It offers integration with mapping service provider Mapbox, allowing real-time access to their provided as well as custom geospatial data within a 3D environment. Unity also interfaces easily with recent immersive devices - in our case, we used the HTC Vive HMD fitted out with an SMI binocular eye tracking device, and its supplied controllers for interacting with the map layers and completing task objectives.

Utilizing the large visual space VR affords, the three MCV variations implemented to take advantage of it were:

- a "blitting" system that displays only one layer at a time on a large map view, with users being able to switch between data layers,

- a grid of smaller map views showing all layers, where interaction with one is mirrored across all others, and

- a stack, that similarly to the grid shows all layers, but tilted and stacked vertically above one another.
The novel third system (the stack) presents the layers at the same size and distance as the grid, but due to the tilt the distance between each layer could be reduced - c.f. fig. 2. This also aimed at making these layers appear more coherent, as there is only one dimension in their arrangement - the same points on each map view appear connected by one vertical line.

This tilt did not prove to be detrimental to map comprehension, as the stereoscopic presentation allows the views to appear naturally, like a map laid on a table - the human visual system automatically compensates for this kind of perspective distortion (Vishwanath et al., 2005), and the map legibility was not rated worse in this variation than in the others in the previous work (Spur et al., 2020).

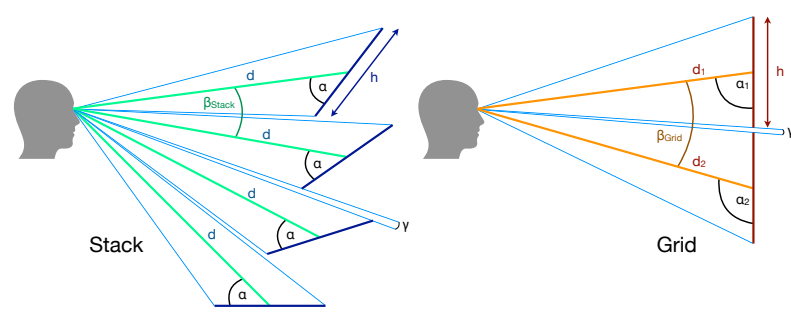

Figure 2. The stack MCV variation displaying layers, compared to the grid: angles between equally-sized layers are smaller, reducing the eye travel required between layers.

All variations were presented in the same virtual room, at a distance and scale that made them appear perceptively equivalent to one another, as determined through pre-testing. The controllers, tracked in six dimensions in space allowed the user to zoom and pan the map layers (coordinated across each view), and interact with a spatial interface to proceed with their tasks.

\section{USER STUDY}

The formal user study was designed with a number of goals:

1. to determine the differences in how users perceived each MCV variation through questionnaires after tasks and analyzing eye tracking data,

2. to see if these immersive MCVs could be used in an actual participatory study in questions regarding urban design, and if the choice of variation had an impact on the quality of the answers participants provided, and

3. the goals of the urban design study:

(a) to assess the knowledge and competence of participants regarding public lighting,

(b) asking them to decide on which parts of the city could benefit from reducing public illumination, and

(c) the importance participants give to the topics of mobility, of respect for the night sky, of energy conservation, and of easy access to nocturnal activities when making these choices.

In order to bring the participants insights to their task in goal $3 \mathrm{~b}$, the topics given in goal $3 \mathrm{c}$ were illustrated as four separate layers of geospatial data, shown in figs. 4 and 5:

- energy consumption (EC) of each individual lamp, visualized as a heat map ranging from blue to red, 
- Light pollution (LP) caused by public lighting, as seen in from an aerial orthophoto taken at night,

- transportation lines and stops active during the night (NT), with colors and shapes matching those of the local transportation maps, and

- points of interest (POI) operating at night, such as restaurants and bars.

These data layers are particularly well-suited to an MCV approach instead of superimposing them on one view, as most of their information lies along streets and overlaps, which would lead to visual clutter and occlusion.

We divided the available data into twelve regions, and chose three candidate areas in each - thus forming twelve scenarios, in each of which our 26 participants (demographics illustrated in fig. 7) were tasked to select the one candidate they considered most in need of reduction of public lighting; example regions are shown in figs. 4 and 5. Special care was taken to make each set of three candidates as comparable as possible from a geometric point of view - three roundabouts, or three crossroads, etc. - to draw attention to their differences in the superimposed data layers, and lessen the impact of their physical characteristics.

After an introduction to the study, three scenarios were used to introduce the three different MCV systems in a tutorial which also reiterated the significance of each data layer (EC, LP, NT, POI). The remaining nine scenarios were randomly assigned to each of the three systems, and the participants were asked to fill out questionnaires related to usability after completing each task, as well as give a written explanation of their choice.

The map layers could be navigated using the controllers, to zoom and pan the views within boundaries close to the three candidate areas - the movement of the controllers was linked to the maps when a button was pressed: parallel movement results in panning, and perpendicular in scaling; as if pulling the map in closer, or pushing it away. The visibility of the candidate outlines could be varied, and a marker could be placed that is mirrored across all views to help with orientation.

Goal 1 of the study was achieved in the previous work's analysis (Spur et al., 2020), establishing a link between user characteristics to their preferences when they rated the MCV variations in terms of legibility, ease of use and visual design. These results were quite polarized, in that the participants were found to split almost evenly in three groups that each preferred one of the variation strongly. To investigate the broader question in goal 2 , we focus in this work on goal 3 , as the extent to which the questions from urbanism can be answered using these MCVs should speak to their usefulness in this endeavor.

\section{RESULTS}

We measured the relative time participants were looking at each layer during their tasks by tracking their eye movement and raycasting against the $\mathrm{MCV}$ windows, discarding the moments when an interface element, such as information overlay was being interacted with. This measurement gave us an indication of attention a participant payed to individual layers of geospatial data during a task.
The other key measure for this study was extracted by analyzing the written free-form answers of each participant for each scenario, explaining the reasons behind their choice of candidate areas in that scenario. We counted the instances a particular data layer was mentioned or referenced in these explanations, as a measure of how important that layer's information was to the subject in that decision.

Tallying these measures up over each participant's answers (fig. 3 for each scenario in percentages and absolute numbers for each candidate allowed for insights into which areas elicited which responses, and how they were argued. Similarly, we used this data to analyze how individual participants reacted (fig. 6).

Analysis of variance shows that these measures vary extremely from person to person ( $p<0.01$ for all data layers), as is expected, and slightly but still significantly from scenario to scenario (tab. 1). Gaze was highly affected, especially on the transport (NT) and POI layers. The systems used to display the data however had no statistically significant effect on either of these.

\begin{tabular}{|c|c||c|c|c|c||c|}
\hline Meas. & Variable & LP & EC & NT & POI & Total \\
\hline \multirow{3}{*}{ Gaze } & System & 0.15 & 0.79 & 0.12 & 0.22 & 0.40 \\
& Scenario & 0.21 & 0.31 & $\mathbf{0 . 0 0}$ & $\mathbf{0 . 0 1}$ & $\mathbf{0 . 0 2}$ \\
& Sys:Sce & 0.72 & 0.98 & 0.10 & 0.62 & 0.79 \\
\hline \multirow{3}{*}{ Mnt. } & System & 0.47 & 0.21 & 0.92 & 0.96 & 0.90 \\
& Scenario & $\mathbf{0 . 0 5}$ & $\mathbf{0 . 0 4}$ & 0.09 & 0.94 & 0.85 \\
& Sys:Sce & 0.96 & 0.07 & 0.58 & 0.62 & 0.77 \\
\hline
\end{tabular}

Table 1. Effects of the system used, the scenario presented, and their interactions on time spent gazing at different data layers and in total, and on the number of times one of the data layers was mentioned (Mnt.) in explaining a choice (p-values).

For the qualitative study on urban lighting, we differentiated the data layers, analyzed the written content of the individual answers, and referenced the choices, attention, and arguments with the actual content of the data layers in the selected areas. To illustrate this process and the level of insight that could be extracted from this study, we present a detailed analysis of contrasting examples of two scenarios and two participants.

\subsection{Scenario analysis}

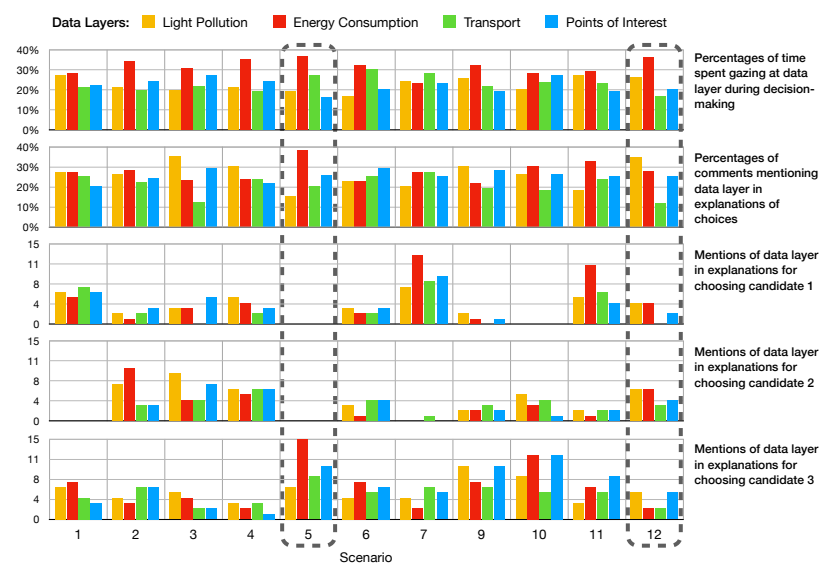

Figure 3. Charts showing data for each scenario: gaze information, candidate choices and layer mentions in explanations.

Scenario 5 (fig. 4) is chosen as an example because it has the strongest unbalanced choices for which area to dim, with most participants (23 out of 26) choosing candidate 5:3, a primary road axis with crossroads, high consumption, high light pollution, low activities nearby, and presence of public transport. 

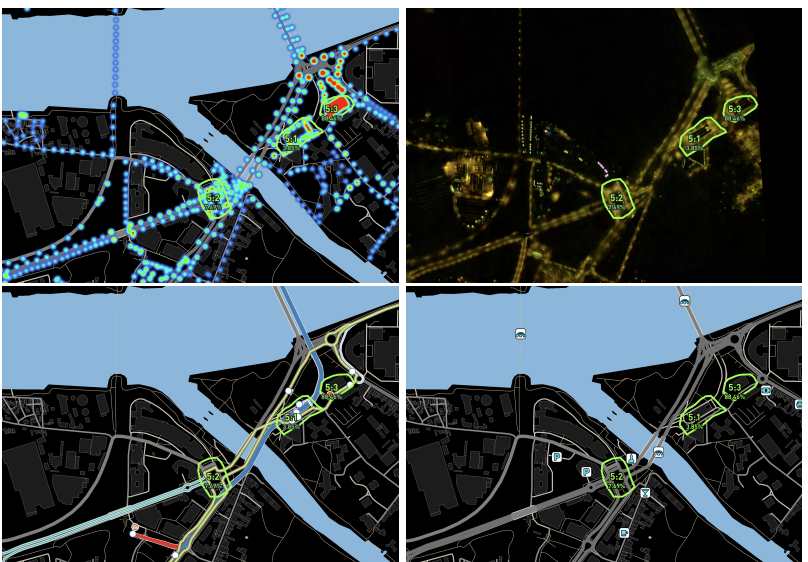

Figure 4. Clockwise from top left: Energy consumption heatmap, light pollution aerial orthophoto, nocturnal points of interest, and nocturnal transport network of scenario 5.

2 participants selected $5: 2$; a primary road axis with a roundabout, high consumption, low presence of night activities, presence of public transport, and moderate light pollution; and only one participant picked 5:1, which contains a tram station, high consumption, low activities nearby, direct public transport, and moderate light pollution.

This candidate 5:3, at the top-right corner of each map (fig. 4), displays a connection with transportation, a high energy consumption, a bit of light pollution and a few night activities. This configuration could be explained by the layout of the city: the candidate is a covered car park close to one of the most important public transportation station. It is brightly illuminated, which explains the importance of the energy consumption, and the roof limits the loss of light to the sky, which explains the dark square on light pollution map (top right).

Layers mentioned in explanations: The 3 participants who chose candidates 5:1 or 5:2 did not mention any of the data they saw when explaining their choices, while the 23 who selected $5: 3$ mostly referred to the energy consumption (38\%), and then in decreasing order activities (26\%), transport (21\%), and finally the light pollution layer $(15 \%)$ when arguing their choice, as seen in fig. 3 .

Layers observed: While selecting candidates in this scenario, the participants spent most of their time (37\%) inspecting the energy consumption layer, and the other layers can be ranked by this metric as follows: transport (28\%), light pollution $(19 \%)$, and lastly the points of interest layer (16\% of time spent gazing at it). The order of how much a layer was inspected differs from how much it influenced the decisions, as measured by the number of mentions in their explanation: while consumption maintains first place, the points of interest are last.

To contrast with scenario 5, scenario 12 (fig. 5) is chosen as an example because it has the most balanced choices among its candidates. 6 of the 26 participants chose candidate 12:1, close to a restaurant with moderate energy consumption, distant from transport and low to no light pollution. 12:2 was chosen by 11 participants, similarly located close to a restaurant, but with localized very high energy consumption, strong light pollution and slightly closer proximity to public transportation. The remaining 9 participants selected the last candidate, similar in all regards to $12: 1$, but with more light pollution.
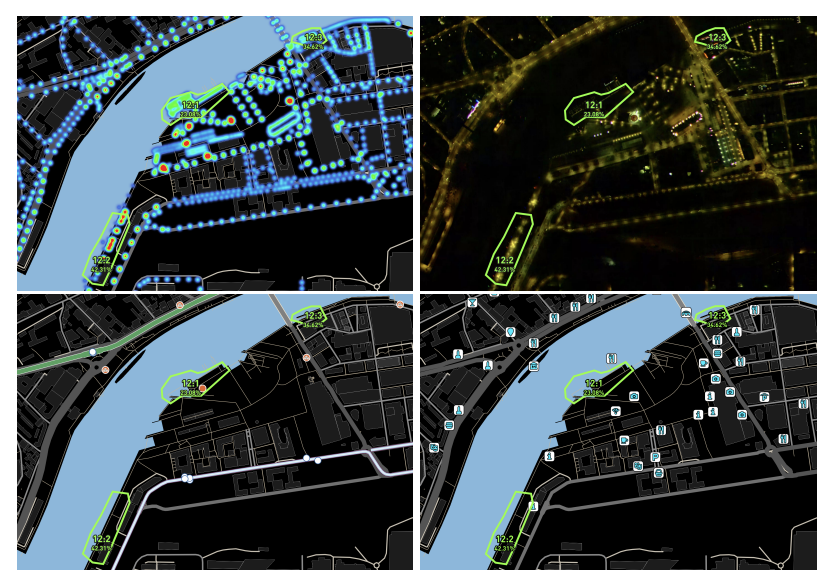

Figure 5. Clockswise from top left: Energy consumption heatmap, light pollution aerial orthophoto, nocturnal points of interest, and nocturnal transport network of scenario 12.

The three candidates are bordering on a river. They are all close to nocturnal activity, mainly restaurants. They have different levels of light pollution and energy consumption, and are more or less close to public transport.

Layers mentioned in explanations: The choices of candidates 12:1 and 12:2 were mostly explained by mentioning the light pollution and energy consumption data (fig. 3). When the candidate 3 was chosen, the two main explanations were mentioning light pollution and points of interest, and to a much lesser degree the other two layers. Overall, this scenario's choices were motivated most by light pollution (35\%), closely followed by energy consumption (28\%) and points of interest (26\%), and to a much lesser degree by the transport network ( $12 \%$ of mentions in the explanations).

Layers observed: As in scenario 5, the most attention was given to the energy consumption layer (36\%), at the expense of the transport network (17\%), which fell behind light pollution $(25 \%)$, and received less attention than the points of interest ( $20 \%$ of time spent gazing at its data layer).

5.1.1 Links between scenario 5 and scenario 12: In scenario 5, the vast majority of participants chose its candidate 5:3. Strongly visible on the energy consumption layer, it seems to have focused participants' attention, although it is directly linked to transport and is not responsible for much light pollution. In scenario 12 , the choice is not so clear: each candidate is on the bank of a river, and some participants in their explanations referred to the introduction text, where fish are mentioned as victims of light pollution. Energy consumption is the most observed data layer, even if it only takes second place in their mentions. Instead, light pollution is the most commented data layer, even if candidates 1 and 2 show less of it in comparison to scenario 5's candidates. Perhaps the dark area of the river created a visual contrast that was driving the choices. In both scenarios, the most consuming area "wins:" candidates 5:3 and $12: 2$ both contain the highest density of energy consumption in their areas.

Whether balanced or not, the ratio of observation percentage, comment percentage, and final candidate selection appears to highlight participants' orientation towards the candidate consuming most energy. This choice could relate to a good understanding of the exercise, the latter being announced as intended to reduce energy consumption and light pollution of public lighting. 


\subsection{Participant analysis}

Part of the purpose of the qualitative analysis of the 26 participants was to observe if their profiles and backgrounds (i.e. age, gender, education and habits with maps, VR experience, video-gaming frequency and familiarity with the city and its maps, fig. 7) have an impact in their choices during the experimentation, in addition to being an indicator as to their preferred MCV variation as determined previously. Also of interest to the urbanist analysis is to investigate links between their attention to the presented data layers as measured by their gaze and the explanations they gave for choosing candidates (fig. 6). As before with the scenarios, we chose two contrasting participants to demonstrate the analysis we did for all.

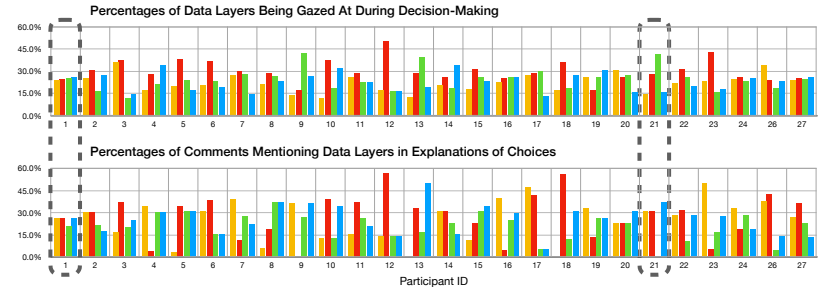

Figure 6. Overview of the gaze and explanations of each participant. Participant 1 and 21 highlighted and analyzed below.
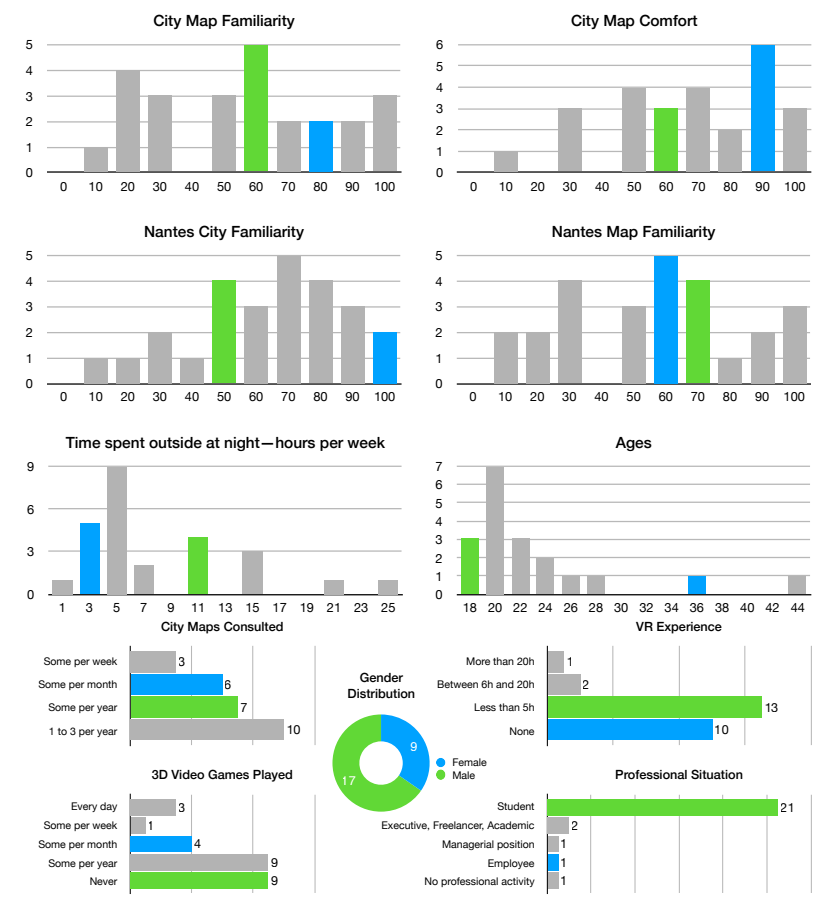

Figure 7. Histograms of participant habits and characteristics;

Participant 1 highlighted in green, participant 21 in blue.

Participant 1 is a 18 year old man, who spends 10 hours a week outside after sunset (fig. 7 green). He is a student and has average familiarity with maps and the presented city (Nantes). His gaze and explanation percentages are well-balanced, in that overall, he spent roughly roughly equal amounts of time observing each data layer, and referenced each of them equally when explaining his choices (fig. 8 top).

Participant 1 regularly observes the criterion of energy consumption, which he describes almost every time as "huge". He is interested in the attractiveness and accessibility of the candidates he selects $(1: 1 ; 2: 2)$, because, according to him, lighting is

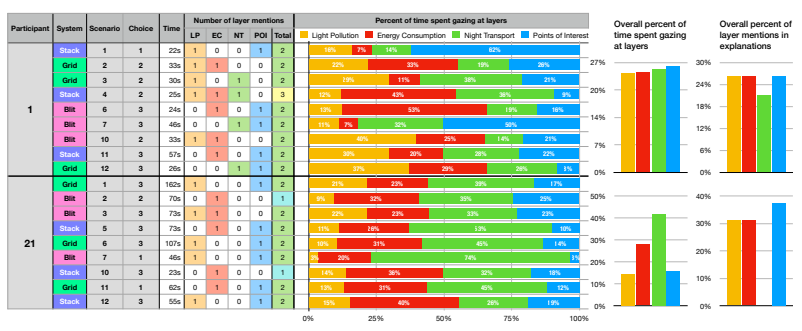

Figure 8. Analysis of two selected participants, showing differences in choices, explanations and visual attention.

synonymous with life, and a place without lighting will therefore be rendered "dead" (6:3). He consolidates his position by choosing for example a candidate he describes as "very attractive and not well lit, which in itself is contradictory" (3:2). Finally, and despite the purpose of the exercise, he proposes twice to add light to the candidates he selects in order to to improve their attractiveness $(2: 2 ; 12: 3)$. For this participant, the notion of public lighting seems decisive in the nightlife that he intends to find in a place, especially if it is easily accessible thanks to a good luminous installation.

Participant 21 is a 38 year old woman, who spends 2 hours a week outside after sunset (fig. 7 blue). She is an employee and has a strong familiarity with maps and the city of Nantes. From her written explanations of the candidate choices we found a good understanding of each data layer, and a strong differentiation between the light produced and the energy it consumes. Her explanations focused mostly on the relation of the lighting to points of activity, and their energy consumption.

Her behavior during the experimentation is characterized as unbalanced, since her gaze and explanation percentages are not aligned (fig. 8 bottom). The participant 21 spent a large amount of time observing the Transport map, but without mentioning it at any time. We can assume that this layer was mostly used for orientation in the city, to place the shown data in known context. Though she mentioned points of interest the most in her answers, that layer is along with the light pollution layer the one she spent the least amount of time inspecting.

She focused on nightlife and energy consumption in the candidates she selected. She placed a lot of importance in reducing light pollution, while concentrating her comments on the subject of activities. Unlike participant 1, she focused on places that are highly lit and yet devoid of nocturnal activities (1:3; $6: 3 ; 7: 1 ; 11: 1 ; 12: 3)$, and therefore believes that these candidates are likely to see their lighting reduced, according with the little activity taking place there. In the same way as candidate 1 , she based this on the importance of energy consumption, which she regularly proposes as the main argument $(2: 2 ; 3: 3$; $5: 3 ; 10: 3 ; 11: 1)$.

\section{DISCUSSION}

The impact of which MCV variation was used had apparently no bearing on the understanding of the data and the answers given, as those depended much more on the data shown and a participant's behavior. It can be seen as a success in making all variations comparably efficient in displaying information, and thus separating the evaluation of their usability against one another from their usefulness for the presented study in urbanism.

The study of individual comments makes it possible to enrich the analysis, showing how two participants can rely on identical 
survey elements to produce different analyses, as shown in the example of participants 1 and 21; the first signalling the absence of lighting as a brake on activity, and the second identifying the absence of activity as a potential for reducing lighting. Differences in the participants' behavior appear to be reflected in their explanations. For the aforementioned participants, nightlife and energy consumption are key elements in their comments.

Light pollution and energy consumption represent the two most visual criteria on the maps, the bright and colorful contrasts of the chosen color palette appearing to engage the participants, while the sparse pictograms in the transport and activity layers offer a relatively fast understanding of the data, as can be seen by the shorter gaze times in fig. 3 .

We could however also observe a differentiation between gaze and comments left by some participants, particularly in the case of the transportation network: since none of the maps were labeled, the familiar bus and tram routes were a way for participants to associate the places shown to them to their mental map of the city. The transport layer was thus not mentioned as often as it was looked at, because it often served a different purpose than judging the need for public illumination. The written explanations corroborate that, as many participants referenced particular locations they remember.

We can note that participants act mainly in the direction of the exercise: they indicate areas of consumption and pollution, and some recall finer details from the introduction text. Congruent with previous studies (Boomsma, Steg, 2014), this shows the possibility to influence an individual's perception according to the quantity and quality of the information they receive. This highlights the need to not just design the visualization systems with care, but also to pay special attention to the introduction and instructions given to participants in these kinds of studies. With this effect, a participatory study could not only be a way to gather information, but an opportunity to educate the participants in these issues. As observed from informal feedback after the test sessions, a large proportion of participants showed eagerness to take part in more studies of this kind, expressing clear enthusiasm for this use of immersive technologies.

\section{CONCLUSION AND FUTURE WORK}

The urban study results are consistent with previous experimentation on public lighting (Deleuil, 2009). To our knowledge, our approach of presenting multilayered data in VR for a public participation study is the first of its kind.

The explanations given by participants show the importance of activities and public transport maps, and indicate the expectations of participants about street lighting: the energy consumption situation may need improvement, but nocturnal public space still has to be made accessible and attractive by artificial light. Since transport and activities seem in a way deeply bound with street lighting concerns, it may be necessary to go further in their representation on maps.

Our study highlights that perception of artificial public lighting seems deeply bound to the background of each participant, and reveals the potential of the notion of user expertise, which could in the future complement the technical and political expertise that today manages public lighting.

Finding no differences in the consistently high quality of the answers given in the participation task depending on which variation of immersive MCV they were presented with leads us to the conclusion that all perform similarly well in the task of soliciting insight and opinion on questions from urbanism involving multiple layers of spatial data. Numerous feedback has expressed enthusiasm for using the virtual environment, showing promise for more studies associated with public practices.

While the specific variation of MCV mainly plays a role in the satisfaction of the participant while using it, its effect is still significant in this regard, and can also impact the speed at which participants can take in and process the displayed information. As this preference is highly personal, a choice of different MCV variations should thus be given for use cases such as the one we presented to avoid frustration and further maximize the engagement immersive environments elicit. Any MCV however enriches the insights that can be gathered by such studies: separating data layers across different views allows for differentiated analysis of attention to each layer, not just in the given answers but especially in the gaze of participants.

We suggest future work to further capitalize on the possibilities that immersive visualization offers. In our study, we presented 2D maps offering a top-down view, and asked questions regarding environments actually experienced from below. Particularly the inclined stack MCV variation could be enriched by a threedimensional city model. All of the presented systems could however extend the MCV metaphor further by providing additional coordinated views of smaller focus areas, such as pedestrian views of individual streets and their buildings, and in the particular domain of light public lighting, a view of the sky.

\section{REFERENCES}

Angulo, A., 2015. Rediscovering Virtual Reality in the Education of Architectural Design: The immersive simulation of spatial experiences. Ambiances. Environnement sensible, architecture et espace urbain.

Berger, M., Bill, R., 2019. Combining VR visualization and sonification for immersive exploration of urban noise standards. Multimodal Technologies and Interaction, 3(2), 34.

Boomsma, C., Steg, L., 2014. The effect of information and values on acceptability of reduced street lighting. Journal of Environmental Psychology, 39, 22-31.

Buyuksalih, I., Bayburt, S., Buyuksalih, G., Baskaraca, A., Karim, H., Rahman, A. A., 2017. 3D Modelling and Visualization Based on the Unity Game Engine-Advantages and Challenges. ISPRS Annals of the Photogrammetry, Remote Sensing and Spatial Information Sciences, 4, 161.

Cavallo, M., Dholakia, M., Havlena, M., Ocheltree, K., Podlaseck, M., 2019. Dataspace: A reconfigurable hybrid reality environment for collaborative information analysis. 2019 IEEE Conference on Virtual Reality and 3D User Interfaces (VR), IEEE, 145-153.

Challéat, S., 2011. La mise en débats des territoires de la lumière. Journée d'études sur Les effets de la participation, GIS Démocratie \& Participation, École des Hautes Études en Sciences Sociales (EHESS), Paris, France.

Chandler, T., Cordeil, M., Czauderna, T., Dwyer, T., Glowacki, J., Goncu, C., Klapperstueck, M., Klein, K., Marriott, K., Schreiber, F., Wilson, E., 2015. Immersive Analytics. 2015 Big Data Visual Analytics (BDVA), IEEE, 1-8. 
Chen, Z., Qu, H., Wu, Y., 2017a. Immersive urban analytics through exploded views. Workshop on Immersive Analytics: Exploring Future Visualization and Interaction Technologies for Data Analytics. Phoenix, AZ.

Chen, Z., Wang, Y., Sun, T., Gao, X., Chen, W., Pan, Z., Qu, H., Wu, Y., 2017b. Exploring the Design Space of Immersive Urban Analytics. Visual Informatics, 1(2), 132-142. http://arxiv.org/abs/1709.08774.

Çöltekin, A., Lokka, I.-E., Zahner, M., Halounova, L., 2016. On the usability and usefulness of $3 \mathrm{~d}$ (geo) visualizations-a focus on virtual reality environments. ISPRS.

Cordeil, M., Cunningham, A., Bach, B., Hurter, C., Thomas, B. H., Marriott, K., Dwyer, T., 2019. Iatk: An immersive analytics toolkit. 2019 IEEE Conference on Virtual Reality and 3D User Interfaces (VR), IEEE, 200-209.

Deleuil, J.-M., 2009. Eclairer la ville autrement, expérimentations et innovations en éclairage public. PPUR.

Dwyer, T., Marriott, K., Isenberg, T., Klein, K., Riche, N., Schreiber, F., Stuerzlinger, W., Thomas, B. H., 2018. Immersive analytics: An introduction. Immersive Analytics, Springer, $1-23$.

Ferreira, N., Lage, M., Doraiswamy, H., Vo, H., Wilson, L., Werner, H., Park, M., Silva, C., 2015. Urbane: A 3D framework to support data driven decision making in urban development. 2015 IEEE Conference on Visual Analytics Science and Technology (VAST), IEEE, 97-104.

Filho, J. A. W., Stuerzlinger, W., Nedel, L., 2019. Evaluating an Immersive Space-Time Cube Geovisualization for Intuitive Trajectory Data Exploration. IEEE Transactions on Visualization and Computer Graphics, 1-1. http://arxiv.org/abs/1908.00580 https://ieeexplore.ieee.org/document/8854316/.

Fonnet, A., Prié, Y., 2019. Survey of Immersive Analytics. IEEE transactions on visualization and computer graphics.

Freitas, S., Catita, C., Redweik, P., Brito, M. C., 2015. Modelling solar potential in the urban environment: State-of-the-art review. Renewable and Sustainable Energy Reviews, 41, 915931.

Gambert, P., n.d. Éclairage public à Nantes : des idées pour payer moins. Ouest France. https://www.ouest-france.fr/paysde-la-loire/nantes-44000/eclairage-public-nantes-des-ideespour-payer-moins- 4854667.

Hurter, C., Riche, N. H., Drucker, S. M., Cordeil, M., Alligier, R., Vuillemot, R., 2018. Fiberclay: Sculpting three dimensional trajectories to reveal structural insights. IEEE Transactions on Visualization and Computer Graphics, 25(1), 704-714.

Karduni, A., Cho, I., Wessel, G., Ribarsky, W., Sauda, E., Dou, W., 2017. Urban space explorer: a visual analytics system for urban planning. IEEE computer graphics and applications, 37(5), 50-60.

Knudsen, S., Carpendale, S., 2017. Multiple Views in Immersive Analytics. Proceedings of IEEEVIS 2017 Immersive Analytics (IEEEVIS).

Kyba, C. C., Hölker, F., 2013. Do artificially illuminated skies affect biodiversity in nocturnal landscapes?
Lobo, M.-J., Pietriga, E., Appert, C., 2015. An Evaluation of Interactive Map Comparison Techniques. Proceedings of the 33rd Annual ACM Conference on Human Factors in Computing Systems - CHI '15, ACM Press, New York, New York, USA, 35733582 .

Lu, A., Huang, J., Zhang, S., Wang, C., Wang, W., 2016. Towards mobile immersive analysis: A study of applications. 2016 Workshop on Immersive Analytics (IA), IEEE, 25-30.

Mahmood, T., Butler, E., Davis, N., Huang, J., Lu, A., 2018. Building multiple coordinated spaces for effective immersive analytics through distributed cognition. 2018 International Symposium on Big Data Visual and Immersive Analytics (BDVA), IEEE, 1-11.

Munzner, T., 2014. Visualization analysis and design. CRC press.

Ortner, T., Sorger, J., Steinlechner, H., Hesina, G., Piringer, H., Gröller, E., 2016. Vis-a-ware: Integrating spatial and nonspatial visualization for visibility-aware urban planning. IEEE transactions on visualization and computer graphics, 23(2), 1139-1151.

Roberts, J. C., 2007. State of the Art: Coordinated \& Multiple Views in Exploratory Visualization. Fifth International Conference on Coordinated and Multiple Views in Exploratory Visualization (CMV 2007), IEEE, 61-71.

Šašinka, Č., Stachoň, Z., Sedlák, M., Chmelík, J., Herman, L., Kubíček, P., Šašinková, A., Doležal, M., Tejkl, H., Urbánek, T. et al., 2019. Collaborative immersive virtual environments for education in geography. ISPRS International Journal of GeoInformation, 8(1), 3 .

Spur, M., Tourre, V., Coppin, J., 2017. Virtually physical presentation of data layers for spatiotemporal urban data visualization. 2017 23rd International Conference on Virtual System \& Multimedia (VSMM), IEEE, 1-8.

Spur, M., Tourre, V., David, E., Moreau, G., Le Callet, P., 2020. Mapstack: Exploring multilayered geospatial data in virtual reality. Proceedings of the 15th International Joint Conference on Computer Vision, Imaging and Computer Graphics Theory and Applications - Volume 3: IVAPP, INSTICC, SciTePress, 88-99.

Trapp, M., Dumke, F., Döllner, J., 2019. Occlusion management techniques for the visualization of transportation networks in virtual $3 \mathrm{~d}$ city models. Proceedings of the 12th International Symposium on Visual Information Communication and Interaction, 1-8.

Vishwanath, D., Girshick, A. R., Banks, M. S., 2005. Why pictures look right when viewed from the wrong place. Nature neuroscience, $8(10), 1401$.

Zhen, W., Yang, L., Kwan, M.-P., Zuo, Z., Wan, B., Zhou, S., Li, S., Ye, Y., Qian, H., Pan, X., 2020. Capturing what human eyes perceive: A visual hierarchy generation approach to emulating saliency-based visual attention for grid-like urban street networks. Computers, Environment and Urban Systems, 80,101454

Zielinska-Dabkowska, K. M., 2018. Make lighting healthier. Nature Publishing Group. 10.1038/d41586-018-00568-7. 